\title{
The Progressive Multifocal Leukoencephalopathy Consortium as a Model for Advancing Research and Dialogue on Rare Severe Adverse Drug Reactions
}

\author{
Ilse S. Peterson • William O. Iverson • Marion T. Kasaian •
}

Maggie Liu

Received: November 20, 2018 / Published online: February 13, 2019

(c) The Author(s) 2019

\begin{abstract}
Progressive multifocal leukoencephalopathy (PML) is a rare but serious disease. Caused by the JC virus (JCV), it occurs in individuals with weakened immune systems and is a potential adverse reaction for certain immunomodulatory drugs. The PML Consortium was created to find better methods to predict, prevent, and treat PML. The Consortium brought together the pharmaceutical industry with academic, regulatory, and patient communities to advance research and dialogue on PML through a not-for-profit, collaborative approach involving a grant program, scientific workshops and conferences, and disease
\end{abstract}

Enhanced Digital Features To view enhanced digital features for this article go to https://doi.org/10.6084/ m9.figshare.7588778.

I. S. Peterson $(\square)$

PML Consortium Secretariat, Consortia

Management Team, Drinker Biddle \& Reath LLP,

Washington, DC, USA

e-mail: ilse.peterson@dbr.com

W. O. Iverson

Translational Sciences, MedImmune, LLC,

Gaithersburg, MD 20878, USA

M. T. Kasaian

Inflammation and Immunology Research Unit,

Pfizer Inc., Cambridge, MA, USA

e-mail: marion.kasaian@pfizer.com

M. Liu

Drug Safety Research and Development, Pfizer Inc., La Jolla, CA, USA

e-mail: Maggie.Liu@pfizer.com awareness efforts. Over nearly a decade, the Consortium contributed to the PML and JCV fields by advancing research, scientific exchange, and awareness of PML. In addition to advancing knowledge and helping to build cross-sector consensus on research priorities, the Consortium's grant program filled a funding gap and brought new investigators into PML and JCV research. Additionally, the Consortium's workshops and conferences created platforms for exchange that drove dialogue on knowledge gaps and future research directions. The Consortium also contributed to the scientific knowledge base with two literature reviews, one on PML treatment studies and a second on $\mathrm{T}$ cell deficiencies as a risk factor for PML and the brain as a site for conversion of harmless JCV into a pathogenic virus. Finally, the Consortium addressed a significant information gap with its disease awareness website for healthcare professionals, patients, and caregivers. Beyond its impact on the PML and JCV fields, the PML Consortium is important because it provides a precedent for how the pharmaceutical industry, academic researchers, patient organizations, and government can work together to address rare diseases, in particular rare adverse events. This kind of collaboration could be replicated to speed progress in addressing other rare diseases and adverse events, with significant potential benefits for the scientific, medical, and patient communities. 
Funding: PML Consortium (PML Consortium, Washington, DC).

Keywords: Multi-stakeholder collaboration; Progressive multifocal leukoencephalopathy; Rare disease; Severe adverse event

\section{INTRODUCTION}

Within the past decade, there has been a proliferation of collaborative initiatives to address scientific and medical challenges. Many new partnerships have been forged to better leverage resources and knowledge in pursuit of improved treatments and care. Cooperative efforts have extended across the private sector, government, nonprofits, and academia and become especially prominent with rare diseases, where limited numbers of patients and limited knowledge about etiology, natural history, and therapeutics often create a greater need to share data and ideas.

One specific area within rare diseases that may benefit significantly from greater collaboration is rare severe adverse drug reactions (ADRs). Without collaboration amongst drug companies and the broader scientific and medical communities, aggregation of data on rare severe ADRs is likely to be limited to regulatory reporting databases. Joint efforts can enable companies to not only share knowledge and data, but also to pool funding and streamline investment in basic and applied research to increase understanding of rare severe ADRs.

The Progressive Multifocal Leukoencephalopathy (PML) Consortium is an early example of collaboration among several pharmaceutical companies to address a rare disease that can occur as an ADR. In the mid-2000s, PML-which had long been recognized as a possible risk for individuals with human immunodeficiency virus (HIV) - unexpectedly emerged as a rare but serious adverse effect associated with several immunomodulatory therapies. PML gained prominence in the mid-2000s because of a series of cases among multiple sclerosis patients receiving natalizumab, although reports of drugassociated PML also emerged in conjunction with other therapies. In 2009, recognition of the serious nature of PML spurred pharmaceutical companies marketing immunomodulatory molecules to come together as the PML Consortium. The PML Consortium's founding members were Biogen Idec, Elan, and Roche; later participants included Pfizer, Bristol-Myers Squibb, MedImmune, and Takeda. The Consortium also had a Secretariat team from Drinker Biddle \& Reath LLP to manage day-to-day operations and provide scientific support and legal advice. Upon formation, the Consortium adopted a vision of finding better methods to predict, prevent, and treat PML associated with immunomodulatory and immunosuppressive treatments. Over the next decade, the Consortium invested significant time, energy, and funds in pursuit of this vision. In doing so, the Consortium also created a model for other efforts to address rare severe ADRs.

\section{FORMATION OF THE CONSORTIUM: PRIORITIZING A COLLABORATIVE, CONSENSUS-BASED APPROACH}

From its early days, the Consortium prioritized knowledge sharing and consensus-driven decision-making as the basis for its activities. The Board of Directors believed this approach would engender a shared sense of purpose and more effective use of expertise and resources. This mindset was reflected in the organization's mission to "understand the pathogenesis of and to reduce occurrence, morbidity, and mortality of PML through a multi-company, not-forprofit, collaborative approach with a shared strategy, investment, data acquisition and analysis and communication with a pre-defined scope and duration." The Consortium's collaborative approach carried through its activities and extended to partnerships with academic and regulatory communities.

\section{THE CONSORTIUM'S ACTIVITIES: DRIVING RESEARCH AND DIALOGUE ON PML}

Consistent with its mission, the Consortium's activities centered on driving research and 
dialogue on PML. The Consortium organized its activities under four Working Groups: the Research, Clinical, Regulatory, and Communications Working Groups. These groups were comprised of subject matter experts from member companies and worked in concert on initiatives to advance the organization's mission. Day-to-day management of activities was supported by the Consortium's Secretariat team, comprised of multi-disciplinary professionals with scientific, project management, legal, and regulatory expertise. This dedicated support helped to ensure that the Consortium made consistent progress against its goals, especially when member company participants were fully occupied by their company responsibilities.

\section{Developing a Research Program to Expand Knowledge of PML}

At the center of the Consortium's activities was a grant program to spur research into PML pathogenesis, risk factors, diagnosis, and management and broaden the field of investigators conducting JCV and PML research. The Consortium's Research Working Group spearheaded this program, which involved broad solicitation of proposals, peer review, and grant administration. Research priorities for each request for proposals were determined collaboratively with the Consortium's Scientific Advisory Board $(\mathrm{SAB})$, a group of academic scientists and clinicians engaged to contribute expertise and increase the program's rigor through participation in the grant review process, which was modeled on the National Institutes of Health's peer-review process.

As part of the research grant program, the Consortium invested $>5$ million dollars in 17 research grants spanning basic virology, immune responses to the causative John Cunningham (JC) virus, pathogenesis, animal model development, and metadata analysis for possible genetic risk factors for PML (see Table 1). To promote knowledge sharing among grantees and Consortium participants, the Working Group organized regular meetings and webinars. Through these events, participating scientists could share findings and discuss questions and areas for future research arising from their work. Overall, this program accelerated research and dialogue on JCV and PML, brought new investigators into the field, and helped to expand the body of literature on these topics.

\section{Addressing Important Clinical Issues}

While the Consortium's research grant program spurred contributions to the scientific literature, the organization also contributed to the literature through its Clinical Working Group. Established to investigate case report data and examine factors that may contribute to PML risk, the Working Group built a clinical database and authored two peer-reviewed literature reviews. The goal of the clinical database was to pool PML case reports from across member companies to create an unparalleled data repository for epidemiologic research. The database was built on Biofortis's LabMatrix platform and combined data from $>400$ confirmed PML cases. However, database use proved challenging because of the incompleteness and lack of consistency in the case reports it contained (the vast majority of which were spontaneously submitted post-marketing reports), which made it difficult to yield meaningful information. Though research from the Consortium's database was never published, the Clinical Working Group did publish two literature reviews. The first, Progressive multifocal leukoencephalopathy (PML): Current treatment options and future perspectives [1], discussed possible drug candidates for prophylaxis and treatment. This included a critical review of PML treatment studies and perspectives for future therapies. The second article, $T$ cell deficiencies as a common risk factor for drug associated progressive multifocal leukoencephalopathy [2], examined evidence for the brain as a site of conversion of harmless JCV into neuropathogenic prototypes. It also proposed a model for PML pathogenesis that emphasized the role of $\mathrm{T}$ cell deficiencies as the key risk factor. 
Table 1 Key research questions addressed by consortium-sponsored research

Research grants funded by the PML Consortium have advanced our understanding of immune responses to JCV and dysregulation of these responses leading to PML. Over 60\% of the world's population has acquired asymptomatic primary infection with JCV, which establishes latency in the kidneys and lymphoid organs. Under conditions of immunodeficiency, the latent virus may become reactivated, with potential to undergo mutations in viral sequence, a change in tropism to the brain, and become pathogenic [3]. As part of its research program, the Consortium addressed the following key questions related to PML and JCV. Citations for related publications are provided where applicable.

Where is the JC virus harbored? One of the key challenges for JCV research is that tissue sites harboring latent or replicative virus have not been comprehensively mapped. Thus, grants were funded to identify tissue reservoirs of JCV and sequence the virus recovered from these sites to determine mechanistic links between mutations in viral sequence and viral latency or tropism [4]. Additional studies examined triggers giving rise to sequence mutations, cellular contributions to hypermutation, and processes leading to defective repair of sequence errors

How does the virus cross the blood-brain barrier? To establish infection in the brain, JCV must cross the bloodbrain barrier (BBB). Model systems were established to investigate whether free virus enters the brain, disrupts the $\mathrm{BBB}$, or is carried across the $\mathrm{BBB}$ in infected cells. $\mathrm{B}$ cells have been proposed as a viral reservoir that would tolerate hypermutation of viral sequences, while acting as a "Trojan horse" to transport virus to the brain. Grants examined JCV isolates from B cell subsets, and possible enrichment of neurovirulent sequences harbored in B cells of natalizumabtreated MS patients

What mechanisms restrict JCV replication in host cells, and what viral factors and host proteins are used by JCV to suppress antiviral signals? $\mathrm{CD} 4+\mathrm{T}$ cells recognize peptides of JCV presented by MHC class II molecules and direct the immune response toward the virus [5], while JCV-specific CD8 $+\mathrm{T}$ cells lyse virally-infected host cells and control viral spread. To understand PML initiation and progression, T cell receptor (TCR) sequences were examined from CD8 + T cells isolated near oligodendrocytes/astrocytes of PML patient brains. The JCV capsid protein VP1 is the main target of T-cell and B-cell immune responses, and VP1 mutations are characteristic of PML. Funded work sought to understand if these mutations reduce binding to host MHC class II molecules, signaling viral escape from host $\mathrm{T}$ cell control. In addition to $\mathrm{T}$ cell and humoral immune responses to JCV, innate antiviral mechanisms restrict JCV growth and spread. In turn, JCV has evolved strategies to suppress these mechanisms. While the field of innate immune sensors has evolved rapidly, the mechanisms that restrict JCV replication in host cells, and the viral factors and host proteins used by JCV to suppress antiviral signals, are largely uncharacterized. Funded work also explored these questions

How does neurovirulant transformation occur? Although JCV sequence insertions, deletions, and rearrangements are associated with neurovirulent transformation and latent virus reactivation, triggers that initiate these changes are not well understood. Funded work examined the process of neurovirulence, with an aim of identifying points for intervention to prevent, halt, or repair sequence alterations $[4,6,7]$. Grants also examined consequences of sequence mutations on JCV replication and viral assembly to understand the mechanism of pathogenesis and identify potential therapeutic targets for treating JCV infection

What factors might help to stratify individuals with increased risk of PML? Treatment with immunomodulatory agents may increase the risk of PML, but the overall risk is low and the vast majority of treated patients do not develop PML [8-11]. The Consortium funded grants to examine host genetic factors that may help to stratify those individuals at greatest risk, and to identify biomarkers that could signal the earliest stages of neurovirulent transformation $[3,12-16]$ 
Table 1 continued

How can animal models be adapted and developed? Investigation of PML pathogenesis and development of therapeutic strategies would be facilitated by a suitable animal model, but is limited by the fact that JCV does not infect non-human species [17]. Models examining related mouse polyomaviruses were adapted to study latency, productive infection, viral spread, and CNS pathology. Experimental humanized mouse models were also developed, which may be susceptible to JCV infection and reproduce aspects of PML-related pathogenesis

\section{Promoting Broader Exchange and Increased Disease Awareness}

In addition to promoting exchange through its research grant program and publications, the PML Consortium sought to stimulate dialogue within the broader scientific and medical communities as well as to provide resources to PML patients and caregivers. The Consortium believed encouraging dialogue with this broader group would yield great value to the PML and JCV communities as a whole and important perspectives on patients' experiences. To this end, the Consortium planned multiple public conferences and launched a website created to share information on PML and the Consortium.

The PML Consortium encouraged broader exchange and knowledge sharing within the scientific and medical communities through two international conferences organized by conference planning committees composed of Working Group, Board, and SAB members. The first was organized with the New York Academy of Sciences and held in New York City in 2013. The second was organized independently by the Consortium and its SAB and held near Gothenburg, Sweden, in 2015. These conferences were open to the public and successful in bringing together Consortium members, grantees, other investigators, and patient representatives from throughout the USA and Europe. They also included regulatory representatives from the US Food and Drug Administration (FDA), the European Medicines Agency (EMA), Health Canada, and the Medicines and Healthcare products Regulatory Agency (MHRA). Proceedings of these events highlight key discussion topics and points of consensus as well as areas for future research $[18,19]$.
Reaching beyond the scientific, medical, and regulatory communities, the PML Consortium also expanded awareness and understanding of PML through a disease awareness website. When the Consortium was formed, there was a substantial amount of misinformation about PML on the internet and relatively little scientifically rigorous information accessible to patient and caregiver populations. To address this, the Consortium created an informative website for healthcare providers, patients, and families, with content tailored to these audiences. Establishment of the website was driven by the Communications Working Group, with content developed in consultation with other working groups and vetted by $\mathrm{SAB}$ members. This website also served as a source for information about the Consortium and its activities, including its grant program and events.

\section{Engaging Regulatory Authorities}

Engagement with regulatory authorities was the fourth primary aspect of the Consortium's activities. Proactively engaging regulatory agencies to share information and obtain input on the Consortium's efforts was especially important given PML's status as an ADR. While the Consortium never focused on any individual products, its goals were critical to member companies and regulatory authorities alike because of their shared responsibility for patient safety. Therefore, the Consortium-through its Regulatory Working Group-built active lines of communication with health agencies beginning in its first year. Over time, the Consortium interacted with the FDA, EMA, Health Canada and MHRA through in-person meetings, webbased presentations, and exchanges at conferences. The EMA, Health Canada, and MHRA 
also became observers on the Consortium's SAB, which provided them with direct insight into the Consortium's research activities. The frequency and depth of these exchanges provided valuable perspective on PML-focused initiatives within all organizations and helped to build concordance in research agendas.

\section{REFLECTING ON THE CONSORTIUM'S ACCOMPLISHMENTS AND AREAS FOR FUTURE WORK}

When the PML Consortium was formed in 2009, PML was a serious concern for many pharmaceutical companies, and one that-because of its rarity-needed to be addressed through a cooperative effort. Over time, however, the ability to stratify PML risk improved, enhanced risk management plans were implemented, and increased awareness helped support earlier intervention. These shifts reduced the urgent drive for clinically meaningful scientific breakthroughs-which are often a longterm, resource-intensive endeavor-pursued by the Consortium's research program. They also hindered the Consortium's ability to attract members to sustain its programs. While still supporting ongoing research grants until their completion, the Board of Directors made a strategic decision to disband the organization after 8 years of intense collaboration.

Looking back on the Consortium's activities, Consortium members, SAB members and grantees, and members of the regulatory science community agree that the Consortium made an important contribution to the PML and JCV fields, demonstrating how a focused, limitedduration collaboration can effectively enable industry to address a critical challenge. Through its grant program, the organization helped to address the funding gap for PML research and propelled expansion of the knowledge base on PML: as of the time of this publication, $>20$ peer-reviewed articles citing support from the Consortium had been published. The grant program also helped to establish new investigators in the field. In 2017, the Research
Working Group analyzed grantee publications over time and estimated that the Consortium had helped to establish at least nine researchers in the field. SAB members and grantees have heralded the program as unique "by virtue of its funding arrangement designed to address a common unmet need" (Leonard Calabrese, 2018, personal communication) and "an extraordinarily helpful initiative, as PML funding for research projects is usually difficult to obtain" (Paola Cinque, 2018, personal communication). In addition, the Consortium's workshops and conferences created important platforms for exchange within the broader scientific, medical, and regulatory communities. One SAB member, Robert Garcea, said of the meetings: "Hearing the best research, and being able to personally communicate with other investigators was invaluable... I believe these meetings generated interest in the field and generated new research" (Robert Garcea, 2018, personal communication).

The Consortium's experiences also highlight challenges that face the JCV/PML field and rare diseases more generally. Despite the relative success of the grant program, the Consortium found attracting innovative, high-quality research proposals challenging, possibly because of the small size of the field or the embryonic nature of the program. Additionally, in spite of progress in addressing knowledge gaps, many open questions on PML and JCV exist. As Kenneth Tyler of the Consortium's SAB observed, "treatment for PML remains essentially non-existent or unproven...[and] a viable animal model for PML that would enable us to analyze pathogenesis, understand risk factors, and test therapeutics has not yet been established. Third, our understanding of host factors that lead to PML remains relatively rudimentary..." (Kenneth Tyler, 2018, personal communication). The challenges the Consortium encountered with its database also highlight how difficult it can be to obtain meaningful data on rare SAEs even when companies are willing to pool data-and how important it is to ensure depth and consistency of data for clinical database efforts to be successful. Finally, the Consortium's experiences underscore how it can be difficult to sustain research when a field 
Table 2 Key factors for successfully managing a multi-stakeholder collaboration on a rare adverse drug reaction

\section{Clear mission and vision}

A clear mission and vision will provide important direction to the collaboration over time, and make it easier to build consensus on specific initiatives and activities

\section{Strong commitment from members}

A strong dedication to the collaboration on the part of the members-and being willing to commit leadership and subject matter expert time as well as financial resources-will help ensure that the collaboration has the motivation, expertise, and funding it needs to succeed

3. Active engagement with academic and regulatory communities

Being able to actively share knowledge with the academic and regulatory communities will be critical for any collaborative effort focused on a rare, severe adverse drug reaction. Each of these groups has important knowledge and perspective that will need to be brought to bear on the challenge at hand

4. Dedicated support to ensure progress

Dedicated support from the Consortium's Secretariat, which included multi-disciplinary professionals with scientific, project management, legal, and regulatory expertise, helped to ensure consistent progress against the Consortium's goals, especially when participants were fully occupied by external priorities

is small, funding comes from limited sources, and clinically meaningful scientific research is a long-term endeavor.

\section{CONCLUSIONS}

The PML Consortium represents an important example of what the pharmaceutical industry can accomplish when it works together and alongside academic experts and regulatory authorities. Though further work is needed, the Consortium helped to spur new research and dialogue on PML at a critical time. In doing so, it also opened the door to new questions and topics that can be examined by other researchers and organizations. More broadly, the Consortium provides a precedent for how companies, academic researchers, and government could work together on other shared challenges, in particular on adverse events (see Table 2). Based on their experience, many members of the Board of Directors and the SAB have expressed that this model should be replicated because it allows researchers from across sectors to work together in ways they could not otherwise. Furthermore, this model has value because it enables more effective use of resources: because it allows participants to align on research priorities and pool expertise and funding to support these priorities, it can streamline research portfolios and avoid redundant efforts. Without this type of collaboration, progress on shared challenges will occur only within individual organizations and likely more slowly than it would in a collaboration.

\section{ACKNOWLEDGEMENTS}

The authors wish to acknowledge the PML Consortium Board of Directors and working group members for their support of this work and contributions to the Consortium; the Consortium's SAB members for their involvement in the Consortium and reflections provided for this paper; the individuals from regulatory agencies who provided input on the Consortium's activities and participated as observers on the Consortium's SAB; and representatives of the patient community who 
shared their perspectives and experiences during Consortium meetings and conferences.

Funding. Funding for development of this manuscript, research referenced herein, article processing, and Open Access publication was provided by the PML Consortium (PML Consortium, Washington, DC).

Authorship. All named authors meet the International Committee of Medical Journal Editors (ICMJE) criteria for authorship for this article, take responsibility for the integrity of the work as a whole, and have given their approval for this version to be published.

Disclosures. Ilse Peterson is an employee of Drinker Biddle \& Reath LLP, the Secretariat for the PML Consortium, which funded this work. William Iverson is an employee of MedImmune, LLC. Marion Kasaian is an employee of Pfizer. Maggie Liu is an employee of Pfizer. The authors have no other relevant conflicts of interest to disclose.

Compliance with Ethics Guidelines. This article does not contain any studies with human participants or animals performed by any of the authors.

Open Access. This article is distributed under the terms of the Creative Commons Attribution-NonCommercial 4.0 International License (http://creativecommons.org/licenses/ by-nc/4.0/), which permits any noncommercial use, distribution, and reproduction in any medium, provided you give appropriate credit to the original author(s) and the source, provide a link to the Creative Commons license, and indicate if changes were made.

\section{REFERENCES}

1. Pavlovic D, Patera AC, Nyberg F, Gerber M, Liu M. Progressive multifocal leukoencephalopathy: current treatment options and future perspectives. Ther Adv Neurol Disord. 2015;8(6):255-73.
2. Pavlovic D, Patel MA, Patera AC, Peterson I. T cell deficiencies as a common risk factor for drug associated progressive multifocal leukoencephalopathy. Immunobiology. 2018;223(6):508-17.

3. Adang L, Berger J. Progressive multifocal leukoencephalopathy. F1000Res. 2015. https://doi.org/10. 12688/f1000research.7071.1.

4. Johnson EM, Wortman MJ, Dagdanova AV, Lundberg PS, Daniel DC. Polyomavirus JC in the context of immunosuppression: a series of adaptive, DNA replication-driven recombination events in the development of progressive multifocal leukoencephalopathy. Clin Dev Immunol. 2013;2013: 197807.

5. Sundqvist E, Buck D, Warnke C, Albrecht E, Gieger $\mathrm{C}$, Khademi $\mathrm{M}$, et al. JC polyomavirus infection is strongly controlled by human leucocyte antigen class II variants. PLoS Pathog. 2014;10(4):e1004084.

6. Wortman MJ, Lundberg PS, Dagdanova AV, Venkataraman P, Daniel DC, Johnson EM. Opportunistic DNA recombination with epstein-barr virus at sites of control region rearrangements mediating JC virus neurovirulence. J Infect Dis. 2016;213(9):1436-43.

7. Johnson EM, Daniel DC. Significance of interviral recombination as novel mechanism for extending viral disease repertoire. Brain Disord Ther. 2016;5(3):127.

8. Schwab N, Schneider-Hohendorf T, Pignolet B, Breuer J, Gross CC, Göbel K, et al. Therapy with natalizumab is associated with high JCV seroconversion and rising JCV index values. Neurol Neuroimmunol Neuroinflamm. 2016;3(1):e195.

9. Borchardt J, Berger JR. Re-evaluating the incidence of natalizumab-associated progressive multifocal leukoencephalopathy. Mult Scler Relat Disord. 2016;8:145-50.

10. Schwab N, Schneider-Hohendorf T, Melzer N, Cutter G, Wiendl H. Natalizumab-associated PML: challenges with incidence, resulting risk, and risk stratification. Neurology. 2017;88(12):1197-205.

11. Schwab N, Schneider-Hohendorf T, Hoyt T, Gross CC, Meuth SG, Klotz L, et al. Anti-JCV serology during natalizumab treatment: Review and metaanalysis of 17 independent patient cohorts analyzing anti-John Cunningham polyoma virus seroconversion rates under natalizumab treatment and differences between technical and biological seroconverters. Mult Scler. 2018;24(5):563-73.

12. Williamson EM, Berger JR. Infection risk in patients on multiple sclerosis therapeutics. CNS Drugs. 2015;29(3):229-44. 
13. Buck D, Hemmer B. Biomarkers of treatment response in multiple sclerosis. Expert Rev Neurother. 2014;14(2):165-72.

14. Schwab N, Schneider-Hohendorf $\mathrm{T}$, Wiendl $\mathrm{H}$. CD62L is not a reliable biomarker for predicting PML risk in natalizumab-treated R-MS patients. Neurology. 2016;87(9):958-9.

15. Schneider-Hohendorf T, Philipp K, Husstedt IW, Wiendl H, Schwab N. Specific loss of cellular L-selectin on $\mathrm{CD} 4(+) \mathrm{T}$ cells is associated with progressive multifocal leukoencephalopathy development during HIV infection. AIDS. 2014;28(5):793-5.

16. Schwab N, Schneider-Hohendorf T, Pignolet B, Spadaro M, Gorlich D, Meinl I, et al. PML risk stratification using anti-JCV antibody index and L-selectin. Mult Schler. 2016;22(8):1048-60.
17. Frost EL, Lukacher AE. The importance of mouse models to define immunovirologic determinants of progressive multifocal leukoencephalopathy. Front Immunol. 2015;5:646.

18. Liu M. E-Briefing on progressive multifocal leukoencephalopathy conference. In: Progressive multifocal leukoencephalopathy conference at the New York Academy of Sciences. 2013 Jun 19-20; New York City, New York (USA): New York Academy of Sciences; 2013.

19. Patera AC, Butler SL, Cinque P, Clifford DB, Elston $\mathrm{R}$, Garcea RL, et al. 2nd international conference on progressive multifocal leukoencephalopathy (PML) 2015: JCV virology, progressive multifocal leukoencephalopathy pathogenesis, diagnosis and risk stratification, and new approaches to prevention and treatment. J Neurovirol. 2015;21(6):702-5. 